\section{Sicherer Opioidgebrauch durch neue Hydrocodon-Formulierung}

\begin{abstract}
Patienten mit mäßigen bis starken Schmerzen benötigen meist Opioide. Hydrocodon in einer missbrauchsgeschützten Retardformulierung ist sicher und wirksam, die Inzidenz von inadäquatem Gebrauch gering. Das ist das Ergebnis einer neue Studie.
\end{abstract}

$\mathrm{M}$ äßige bis starke Schmerzen mindern Lebensqualität und psychisches Wohlergehen, beeinträchtigen den Schlaf, partnerschaftliche Beziehungen und die berufliche Tätigkeit. Leitlinien empfehlen in diesen Situationen den Einsatz von Opioiden wie Hydromorphin, Hydrocodon oder Oxycodon, bevorzugt in retardierten Formulierungen, um die Compliance der relativ kurz wirksamen Substanzen zu verbessern. Nachteil der Retardformulierungen sind hohe Wirkstoffkonzentration, die das Risiko für missbräuchliche Anwendungen etwa durch Injektion oder Dosedumping in Kombination mit Alkohol erhöhen. Arzneimittelbehörden in aller Welt forcieren daher die Entwicklung von missbrauchssicheren Opioidtabletten. Eine solche Hydrocodon-BitartratFormulierung mit protrahierter Freisetzung (ER) hat die Firma Teva vorgelegt.
Die Tabletten sind manipulationssicher und zeigten in Phase-I-Studien die erwartete Dosis-Wirkungs-Beziehung für Hydrocodon. In einer randomisierten doppelblinden Phase-III-Studie sollte die schmerzstillende Wirksamkeit dieser neuen, zweimal täglich anzuwendenden Tabletten bei insgesamt 294 Patienten mit mäßigen bis starken Arthroseschmerzen oder Schmerzen des tiefen Rückens im Vergleich zu Placebo untersucht werden.

Beim primären Zielkriterium, der Veränderung der durchschnittlichen Schmerzintensität vom Behandlungsbeginn bis zur Behandlungswoche zwölf, jeweils wöchentlich gemessen, ergaben sich keine signifikanten Unterschiede zwischen Hydrocodon ER und Placebo. Dieses zunächst überraschende Ergebnis relativierte sich allerdings in einer Posthoc-Analyse bei Ausschluss der nied- rigsten applizierten Verumdosis von $15 \mathrm{mg}$ - der Verum-Placebo-Unterschied war mit $p=0,032$ signifikant. Signifikant mehr Patienten aus der Placebogruppe berichteten über einen 33- bzw. $50 \%$ igen Anstieg der durchschnittlichen Schmerzintensität $(\mathrm{p}<0,05)$ und die Intensität der wöchentlichen Schmerzspitzen in Woche 12 waren unter Hydrocodon ER signifikant niedriger $(\mathrm{p}=0,026)$. Dementsprechend war der zusätzliche Gebrauch von weiteren Schmerzmitteln in der Verumgruppe auch niedriger als unter Placebo. Die Inzidenz von versuchtem Abusus war gering, die Nebenwirkungsrate in beiden Gruppen vergleichbar.

Fazit: Hydrocodon ER als missbrauchsgesicherte Retardformulierung ist wirksam und sicher bei Patienten mit mäßigen bis starken Schmerzen. Möglicherweise ist der analgetische Effekt der niedrigsten Dosis mit $2 \times$ täglich $15 \mathrm{mg}$ für eine Analgesie bei diesen Patienten nicht ausreichend. Barbara Kreutzkamp

Hale ME et al. Efficacy and tolerability of a hydrocodone extended-release tablet formulated with abuse-deterrence technology for the treatment of moderate-to-severe chronic pain in patients with osteoarthritis or low back pain. J Pain Res 2015;8:623-36

\section{Achtsamkeitstraining lindert Rückenschmerzen}

Die Achtsamkeits-basierte Stressreduktion ist vor allem in den USA ein populäres Programm, das primär zur Stressbewältigung entwickelt wurde. Neuen Erkenntnissen zufolge, scheint es auch bei chronischen Rückenschmerzen gute Effekte zu zeigen.

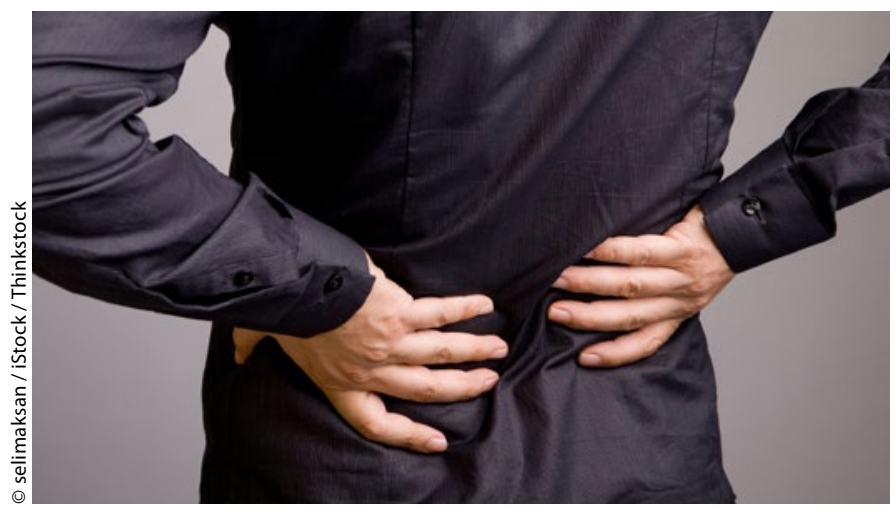

Eine verbesserte Körperwahrnehmung kann helfen, chronische Rückenschmerzen zu verringern.
$D^{i}$ Wirksamkeit von Achtsamkeitsbasierter Stressreduktion („mindfulness-based stress reduction“, MBSR) bei chronischen Rückenschmerzen wurde in einer randomisierten Studie im Vergleich zu einer kognitiven Verhaltenstherapie („cognitive behavioral therapy“, CBT) und zur üblichen Therapie untersucht. Studienteilnehmer waren 342 Erwachsene im Alter zwischen 20 und 70, die im Mittel schon seit sieben Jahren an unspezifischen Kreuzschmerzen litten. 113 sollten ihre bisherige Therapie fortsetzen, 116 zusätzlich eine MBSR und 113 eine CBT absolvieren. MBSR sowie CBT bestanden aus einem achtwöchigen Gruppentraining für jeweils zwei Stunden pro Woche. Die Patienten wurden außerdem angeleitet, die Übungen zuhause weiterzuführen. MBSR beinhaltete das Trainieren von Körperwahrnehmung (BodyScan), Yoga und Meditationen, mit dem 